\title{
Principales limitaciones del empresarismo que afectan el desarrollo económico y social del país
}

\author{
Adriana Giraldo Múnera* \\ Giovanni Bedoya Bermudez** \\ Carlos Mario Vargas Restrepo***
}

\section{RESUMEN}

La creación de empresas, ha adquirido relevancia en los últimos años al convertirse

en el instrumento para contrarrestar los indices de desempleo en el país. De ahi los esfuerzos del estado colombiano por promover la actividad emprendedora.

Las operaciones empresariales inciden directamente en el desarrollo económico del país, pero Colombia no logra adecuados niveles en este sentido, en razón a los bajos estándares de innovación tecnológica, de investigación y desarrollo, que redundan en productos con poco valor agregado y por tanto en limitaciones de productividad y de

competitividad de las empresas.

Finalmente, existe otro factor que repercute de manera negativa en el desarrollo económicosocial del pais: la informalidad de muchas empresas, configurada en prácticas ilegales. Aspectos serán analizados con algún grado de detalle en este documento.

\section{Palabras Clave \\ Desarrollo económico \\ Empresa \\ Innovación \\ Investigación y desarrollo}

\section{Introducción}

$E_{1}$ nivel de desarrollo económico y social de un país, depende en gran medida de la dinámica emprendedora que ostente en su interior y de los esfuerzos conjuntos, del sector público y privado, por promover la cultura emprendedora, sobretodo la que contiene altos estándares de innovación, investigación y desarrollo. En este sentido, el presente artículo pretende hacer una

* Candidata a Maestría en Gestión de Organizaciones Universidad Quebec-EAN. Especialista en Comunicación para el Desarrollo Universidad de Navarra - España. Especialista en Mercadeo de EAFIT Comunicadora Social UPB. Subdirectora Administrativa y Asistente del Director de la Caja de Compensación Familiar Comfenalco Antioquia.

** Candidato a Maestría en Gestión de Organizaciones Universidad Quebec-EAN. Especialista en Gerencia Financiera y Administrador de Empresas de la Universidad Pontificia Bolivariana. Profesor de Cátedra Universidad Ceipa. Consultor Empresarial.

*** Candidato a Maestría en Gestión de Organizaciones Universidad Quebec-EAN. Especialista en Gestión Tributaria Universidad de Antioquia. Contador Público Universidad Católica de Oriente. Docente de tiempo completo Universidad Católica de Oriente. Docente de cátedra Universidad de Antioquia. Asesor de empresas, públicas y privadas, en temas contables, financieros, tributarios y de revisoría fiscal. 
reflexión en torno a las limitaciones que experimentan los empresarios al momento de crear nuevos negocios, e inclusive aquellos que ya tienen empresas establecidas y cómo dichas limitaciones repercuten directamente en el desarrollo económico y social de Colombia.

El trabajo está estructurado en tres partes. En la primera, se realiza una breve contextualización del tema de creación de empresas en Colombia, analizando su situación frente a los demás países objeto del estudio del Global Entrepreneurship Monitor -GEM. La segunda parte, plantea la problemática general que enfrentan los empresarios nacientes y establecidos: tamaño de las empresas, insuficiente infraestructura para investigación y desarrollo, escasos procesos de innovación, producción de bienes y servicios sin elementos diferenciadores, etc. Estos hechos se convierten de alguna manera en obstáculo para el desarrollo económico-social del país.

En la tercera parte, se describe un aspecto que ha limitado el desarrollo de las empresas y del país: la informalidad desdelaque operanalgunos empresarios, manifestada en prácticas ilegales y evasoras. Finalmente, se plantean algunas conclusiones que sintetizan la situación problemática que rodea el empresarismo y que tiene su repercusión directa en los niveles de desarrollo económico y social de Colombia.

\section{Abstract}

The creation of companies have become an important tool to face unemployment rates in Colombia. For this, the Colombian government is making a special effort to develop an entrepreneurial spirit.

The entrepreneurial actions impact the economic development of the country, but Colombia doesn't yet reach the required levels due to the low standards in technological innovation, as well as in research and development that result in products showing poor added value and limitations in productivity and competitiveness.

Finally, there is another factor that deeply interfere in the development of the country, company informality, based on illegal practice. This aspect is also analyzed in this paper.

\section{KEy WORDS}

Economic development

Company

Innovation

Research and development 


\section{Contextualización}

Los

os análisis preliminares del informe del Global Entrepreneurship Monitor GEM- 2007 para Colombia, permiten visualizar el acentuado crecimiento de las nuevas empresas en el país, lo que sin dudas resulta un hecho que debe considerarse como positivo para el desarrollo económico del mismo. En efecto, durante el año 2007, Colombia se sitúo en el segundo lugar de los países de mediano y bajo ingreso en el cual se crean más empresas, después de Perú.

Para el año 2008, el estudio GEM hizo otra clasificación de los países dividiéndolos en economías basadas en los recursos, economías basadas en la eficiencia y economías basadas en la innovación. Colombia ocupó igualmente la misma posición después de Bolivia, pero con una connotación especial, consistente en que se ubica dentro del grupo de países cuya economía se basa en los recursos ${ }^{1}$, es decir, sistemas en los que los recursos se consideran disponibles de alguna manera y se caracterizan por soportar gran parte de su economía en actividades agrícolas, pecuarias, etc. y muchas veces sin procesos tecnificados.

Las diferencias con los países de otros escenarios saltan a la vista: en el estadio de la eficiencia, se ubican aquellos países que logran niveles de competitividad fabricando sus bienes y servicios de mejor calidad, adquiriendo tecnología para mejorar en investigación y desarrollo, etc, mientras que en el estadio de la innovación, los países logran desarrollar su propia tecnología y sus propios procesos; a partir de ahí mejoran ostensiblemente sus bienes y servicios porque les inyectan investigación y desarrollo, lo que se traduce en agregación de valor y con esto, diferenciación y posicionamiento en los diferentes mercados.

En la misma clasificación de Colombia aparecen además de Bolivia, los siguientes países: Angola, Ecuador, Egipto, India, Irán y Bosnia. En el grupo de países con economías basadas en la eficiencia, Perú es quien ocupa el primer puesto de creación de empresas en el año 2008 y el menor nivel de creación de empresas lo alcanza Rusia. Entre estos dos extremos, aparecen en orden descendiente, los siguientes países: República Dominicana, Argentina, Jamaica, Macedonia, Chile, México, Brasil, Uruguay, Sudáfrica, Serbia, Croacia, Hungría, Letonia, Turquía y Rumania.

En el otro grupo, en los países que basan su economía en la innovación; Estados Unidos es el país donde más empresas se crearon durante el año 2008 y su nivel sigue estando por debajo del

${ }^{1}$ Informe GEM Chile 2008. P 31. 
alcanzado por Colombia. Le siguen Islandia, República de Corea, Grecia, Noruega, Irlanda, Finlandia, España, Israel, Eslovenia, Reino Unido, Francia, Japón, Holanda, Italia, Dinamarca, Alemania y Bélgica; país de este grupo donde menos negocios nacieron. Como se anotó, ninguno de los países de este último grupo, ni del de los que tienen su economía basada en la eficiencia -con excepción de Perú- alcanza el nivel de creación de empresas colombiano durante un año (ver gráfica 1).

Gráfica 1. Actividad emprendedora en etapas iniciales (TEA) para 43 países en 2008, por fase de desarrollo económico, con intervalos de confianza del $95 \%$

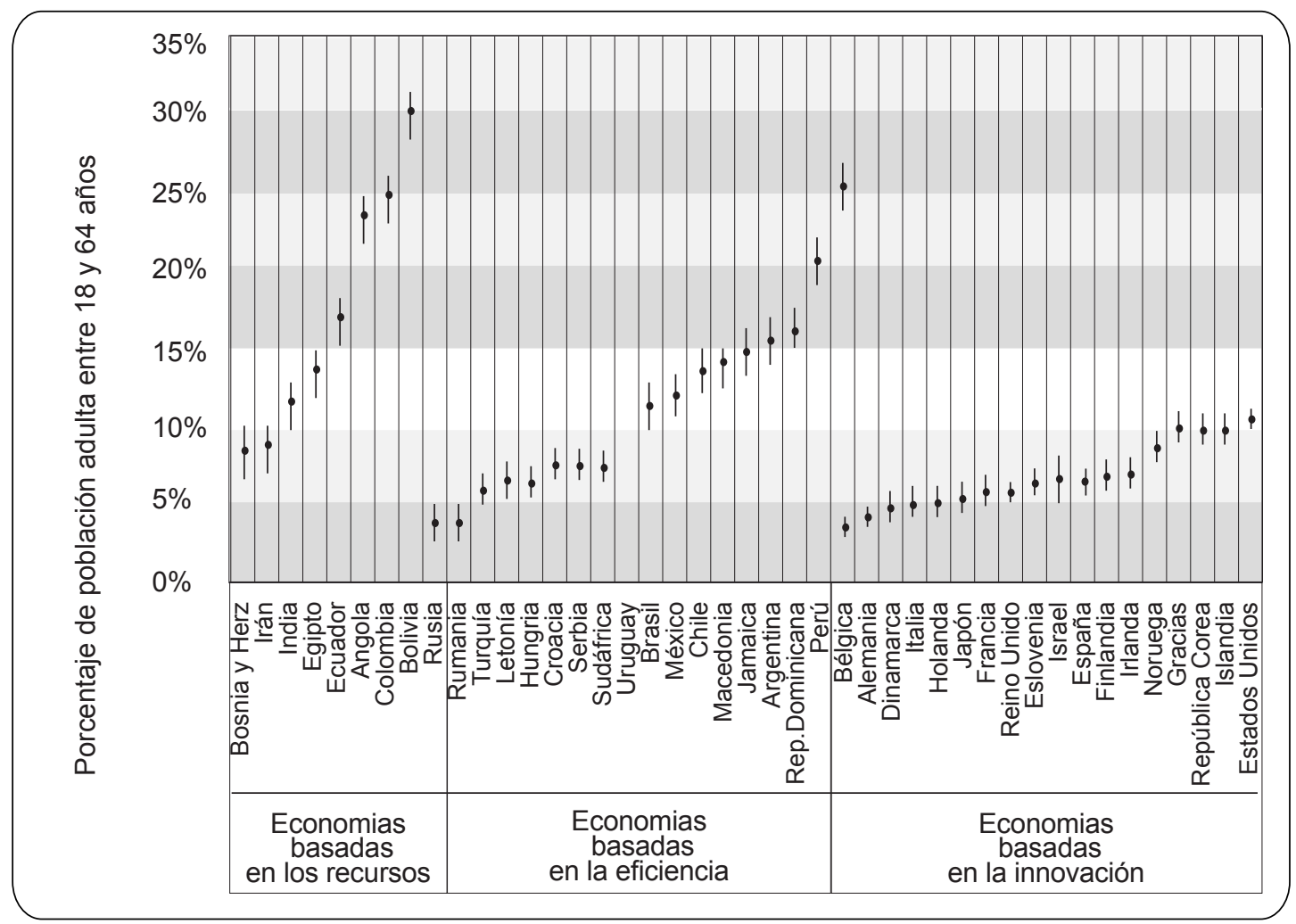

Fuente. Informe GEM Chile 2008.

A partir de esta situación se deduce que el nivel de desarrollo de un país es inversamente proporcional a la creación de empresas, ello es, a mayor crecimiento y desarrollo de la economía, menor creación de empresas, y viceversa. De este contexto surge igualmente otra tesis: en los países desarrollados, las nuevas empresas surgen por oportunidades del mercado, mientras que en los países 
emergentes y/o en desarrollo, las nuevas empresas surgen por necesidad, es así como el estudio GEM Colombia 2007 señala que en el país, "un porcentaje muy alto de la población crea empresas, pero no porque sea su mejor opción, sino porque no tiene otra"(GEM, 2007).

En el primer caso por tanto y gracias al entorno económico que rodea a las nuevas empresas, el componente de conocimiento, tecnología e innovación, es muy superior al que pudieran registrar las nuevas empresas de los países de menor desarrollo económico; de ahí las ventajas competitivas y comparativas que logran en sus mercados locales e internacionales. Rodrigo Varela indica que "en los países desarrollados se tiene claridad sobre la relación entre el desarrollo económico y la innovación tecnológica, razón por la cual se dedican sumas significativas al desarrollo de infraestructuras adecuadas para la investigación, el desarrollo y la innovación tecnológica".

Con lo anterior no se quiere expresar que sea del todo algo negativo; por el contrario, la creación de empresas -así sea en estas condiciones- ha permitido de alguna manera dinamizar la economía de estos países específicamente Colombia y a disminuir los niveles de desempleo. "Según ACOPI y el Ministerio de Comercio, Industria y Turismo, las PyMEs representan cerca del 34\% del valor agregado, generan el $37 \%$ de la producción, el $77.1 \%$ del comercio, el $68.7 \%$ de los servicios, el $73 \%$ del empleo urbano, el $50 \%$ del empleo industrial, el $30 \%$ de la inversión neta, el $43.5 \%$ del consumo industrial, el $32 \%$ de las exportaciones no tradicionales, el $46 \%$ de las importaciones; cifras que reflejan la profunda importancia de las PyMEs en la economía nacional"(Calle, 2009). Sin embargo, si resulta pertinente, diseñar desde los sectores público y privado; políticas, programas y mecanismos que permitan a los empresarios colombianos, establecidos y nacientes, consolidar sus procesos productivos y agregar a los mismos componentes de conocimiento, tecnología, investigación e innovación.

Rodrigo Varela expresa esta situación de manera clara al señalar que "cada vez se hace más notable, a escala mundial, la relación directa entre la dinámica económica y el nivel de innovación de las empresas, las regiones y los países. La realidad es que la competitividad y el desarrollo están basados en las innovaciones tecnológicas, sean en tecnologías blandas o en tecnologías duras, con tecnologías de punta o con tecnologías tradicionales. La innovación establece las ventajas competitivas y por ello es el alma de todo el proceso empresarial". 


\section{Problemática que rodea la actividad emprendedora en el país}

S de nuevas empresas en el país resultan alentadoras, también preocupan las estadísticas sobre la liquidación y cierre de otras tantas que mueren en el intento o peor aún, de algunas que se extinguen después de haber iniciado operaciones. De hecho, el informe GEM 2007 indica que en Colombia, un promedio aproximado del $15 \%$ de las personas consultadas, cerró algún tipo de empresas(GEM, 2007). Los factores que explican esta funesta situación pueden ser muy variados, entre los principales se destacan: falta de planeación del negocio a través de un adecuado plan de empresa, cuantiosas sanciones por operar desde la informalidad, carencia de ventajas competitivas y comparativas por sus bajas inversiones en innovación tecnológica, e insuficientes infraestructuras para investigación y desarrollo, entre otras.

Este factor de competitividad adquiere relevancia y por ello se analizará desde dos ópticas: tamaño de las empresas existentes y de las que se crean; y los medios bajo los cuales desarrollan sus actividades económicas.

En primer lugar, la mayor parte de las empresas del país son pequeñas, es decir se crean muchas empresas pero la mayoría de ellas -por no decir todas- son micro y pequeñas, o a lo sumo medianas. De acuerdo con datos del Ministerio de Comercio, Industria y Turismo ${ }^{2}$, un porcentaje extremadamente elevado de las empresas del país, el 99\%, son micro, pequeñas y medianas (MiPyMEs).

Valdría la pena en este punto aclarar los conceptos o los criterios en virtud de los cuales se catalogan a las empresas como medianas o pequeñas; los cuales están contenidos en el Artículo Primero de la Ley 905 de $2004^{3}$ que definen la clasificación de acuerdo con el número de empleados vinculados y de acuerdo con el monto de sus activos totales, independiente de la actividad o sector económico al que pertenezcan, así:

\footnotetext{
MINISTERIO DE COMERCIO, INDUSTRIA Y TURISMO. Importancia y dificultades de las MIPYMES. En: http://www. mincomercio.gov.co/eContent/NewsDetail.asp?Source=ExpiredNews\&ID=1294\&IDCompany=1. (Consulta: 16 de agosto de 2009). ${ }^{3}$ CONGRESO DE LA REPÚBLICA. Ley 905 de 2004 (Diario oficial No 45.628 del 02 de agosto de 2004). "Por medio de la cual se modifica la Ley 590 de 2000 sobre promoción del desarrollo de la micro, pequeña y mediana empresa colombiana y se dictan otras disposiciones".
} 
Tabla 1

Clasificación de las micro, pequeñas y medianas empresas

\begin{tabular}{|c|c|l|}
\hline Clasificación & $\begin{array}{c}\text { Numero de } \\
\text { empleados }\end{array}$ & $\begin{array}{c}\text { Monto total } \\
\text { de activos }\end{array}$ \\
\hline Microempresa & Hasta 10 & $\begin{array}{l}\text { Inferiores a } \\
\text { 500 SMMLV } \\
\text { De este valor } \\
\text { se excluye la } \\
\text { vivienda de } \\
\text { habitación del } \\
\text { empresario }\end{array}$ \\
\hline $\begin{array}{c}\text { Pequeña } \\
\text { empresa }\end{array}$ & Entre 11 y 50 & $\begin{array}{l}\text { Entre 501 y } \\
\text { menos de } \\
5.000 \text { SMMLV }\end{array}$ \\
\hline $\begin{array}{c}\text { Mediana } \\
\text { empresa }\end{array}$ & Entre 51 y 200 & $\begin{array}{l}\text { Entre } 100.000 \\
\text { a 610.000 } \\
\text { UVT }\end{array}$ \\
\hline $\begin{array}{c}\text { Grande } \\
\text { empresa }\end{array}$ & Mas de 200 & $\begin{array}{l}\text { Más de } \\
610.000 ~ U V T\end{array}$ \\
\hline
\end{tabular}

En segundo lugar y ligado a la situación anterior, el hecho de que la estructura empresarial esté soportada en pequeñas y medianas empresas, dificulta los procesos de industrialización y de producción en economías de escala, lo que a su vez conlleva a fuertes limitaciones para enfrentar la competencia y por supuesto a dificultades respecto a las condiciones de permanencia en el mercado.

Estos hechos sumados a algunas otras características de este tipo de empresas, hacen que sean un tanto vulnerables a las variaciones que les pueda presentar el entorno (económico, social, político, legal, etc.) y por tanto los riesgos que deben afrontar son constantes.

La misma fuente citada del Ministerio de Comercio, Industria y Turismo, identifica como dificultades de las MiPyMEs: "1) limitaciones técnicas y competitivas que imponen las escalas de producción, 2) dificultades en la identificación y acceso a la tecnología adecuada, 3) falta de asociatividad empresarial, 4) carencia de directivos con capacidad gerencial y pensamiento estratégico y 5) dificultad de cimentar la articulación del sector con la gran empresa y con los sistemas de compras estatales" ${ }^{\prime}$.

Por su parte, el Consejo Nacional de Política Económica y Social -CONPES ${ }^{7}$ -estableció como debilidades de las microempresas y de las PyMEs, las siguientes: "sus altos niveles de informalidad; sus bajos niveles de asociatividad; la estrechez de los mercados a los que dirigen sus productos; el bajo nivel económico y de formación de sus recursos humanos; el limitado acceso al sector financiero; su baja capacidad de innovación; el bajo uso de tecnologías de información y comunicaciones (TICs); los problemas para la comercialización

\footnotetext{
Salarios mínimos mensuales legales vigentes

Unidad de Valor Tributario. Creada por el articulo 51 de la Ley 1111 de 2006. Para 2008, el valor de la UVT era de $\$ 22.054$ Ministerio de Comercio, Industria y Turismo. Importancia y dificultades de las MIPYMES. En: http://www.mincomercio.gov.co/ eContent/NewsDetail.asp?Source=ExpiredNews\&ID=1294\&IDCompany=1. (Consulta: 16 de agosto de 2009).

7 Consejo Nacional de Política Económica y Social. Documento CONPES 3484 de 2007 (Política nacional para la transformación productiva y la promoción de las micro, pequeñas y medianas empresas. Un esfuerzo público privado). P 3 y 5.
} 
de sus productos y la obtención de insumos y la limitada participación en el mercado de la contratación pública".

Como puede observarse, ambas fuentes guardan total concordancia al definir las características que obstaculizan el pleno desarrollo de este tipo de empresas; situación que tiene incidencia directa en la economía del país, la cual se reciente y no logra alcanzar adecuados niveles de crecimiento y desarrollo.

En este sentido, la oferta de bienes y servicios de la mayoría de empresas colombianas, no poseen características suficientes de calidad, de posicionamiento de marca, de diferenciación y de agregación de valor en tecnología y conocimiento. En este punto radican, como se mencionó anteriormente, fuertes diferencias con los empresarios de los países desarrollados, en donde pueden introducir en sus bienes y servicios todos estos elementos, máxime cuando sus negocios surgen de las oportunidades brindadas por el mercado.

Colombia por el contrario, se ha caracterizado porque la mayoría de negocios se crean por necesidad, como mecanismo para contrarrestar los elevados índices de desempleo, que sumado a la baja capacidad de inversión y al difícil acceso a fuentes de financiación, conducen a los resultados descritos en los párrafos precedentes. El informe GEM 2008 de Chile resalta esta situación, la cual debería analizarse y tenerse en cuenta entre los dirigentes colombianos (públicos y privados), máxime cuando el país austral ha logrado mejores niveles de competitividad que Colombia. En dicho informe se señala que "las variables transferencia de $1+\mathrm{D}^{8}$ ,educación y acceso al financiamiento son los factores con menor evaluación y constituyen las principales barreras para el desarrollo del emprendimiento en Chile"(GEM, 2008). Colombia sin duda, está en peores escenarios que los chilenos, esto se convierte en un claro obstáculo que le resulta desfavorable al crecimiento y desarrollo económico-social del país.

Con todo, no debe desconocerse que las PyMEs se han convertido en un factor que ha promovido el dinamismo del sector productivo formal e informal de la economía, básicamente a través de la generación de empleo. Como lo expresa Hernando Granados ${ }^{9}$, "en la política económica y social este tipo de empresas inciden en la reducción de los niveles de pobreza, la generación de empleo de baja cualificación y en el acceso a la población de bajos recursos

\footnotetext{
Investigación y Desarrollo

9 GRANADOS Cortes, Hernando. El papel de las micro y pequeñas empresas en el desarrollo territorial: Una lectura desde los programas de fortalecimiento empresarial en la ciudad de Medellín. En: Mercatec, revista de la Institución Universitaria Esumer. No 45, Febrero de 2009. P 21.
} 
por productos de difícil obtención". Sin embargo, las PyMEs no han contribuido de manera efectiva al crecimiento $y$ desarrollo económico del país en términos significativos, en razón a que no logran posicionar en el mercado -especialmente en el internacional-, productos con diferenciación en marca y valor agregado, como aspectos que consolidan y hacen fuerte la economía de un estado.

Aunque elgobierno ha realizado esfuerzos, especialmente con la expedición de normas y creación de estímulos (ver cuadro 2), estos no logran aún dar los resultados esperados, quizá porque han sido inmediatistas (de aplicación en el corto plazo) y de políticas de coyuntura. No han sido políticas estructurales que garanticen la permanencia de los nuevos empresarios en el tiempo y en el mercado, así como su productividad y eficiencia en los procesos. De ahí que la dinámica continúa siendo la misma: creación de empresas para hacerle la guerra al desempleo y a la inequidad y no procesos empresariales suficientemente innovadores, sólidos en tecnología, investigación y desarrollo.

Tabla 2. Principales disposiciones normativas que regulan y promueven la actividad emprendedora en Colombia

\begin{tabular}{|c|c|}
\hline Norma & Descripción breve de la norma \\
\hline Ley 79 de 1988 & $\begin{array}{l}\text { Establece el marco normativo general del sector cooperativo: características, } \\
\text { constitución, etc. }\end{array}$ \\
\hline Ley 590 de 2000 & $\begin{array}{l}\text { Introduce el término PyME como tal. Señala los parámetros y características } \\
\text { requeridos para clasificar las empresas en micro, pequeñas o medianas de acuerdo } \\
\text { al número de empleados y activos totales. De igual forma busca fortalecer este sector } \\
\text { incentivando el surgimiento y sostenimiento través de instrumentos de apoyo. }\end{array}$ \\
\hline Ley 905 de 2004 & $\begin{array}{l}\text { Modifica la Ley } 590 \text { de } 2000 \text { sobre promoción del desarrollo de la micro, pequeña } \\
\text { y mediana empresa colombiana y dicta otras disposiciones como la de otorgar } \\
\text { beneficios fiscales en materia de impuestos territoriales con el fin de estimular la } \\
\text { creación y subsistencia de las PyMEs. }\end{array}$ \\
\hline Ley 1014 de 2006 & Llamada Ley de fomento a la cultura del emprendimiento. \\
\hline $\begin{array}{l}\text { Leyes de reformas } \\
\text { tributarias ( } 863 \text { de } 2003 \\
1111 \text { de } 2006)\end{array}$ & $\begin{array}{l}\text { Contemplan incentivos fiscales para las PyMEs: acceso a contratos de arrendamiento } \\
\text { financiero, bajo la modalidad de leasing operativo. }\end{array}$ \\
\hline $\begin{array}{l}\text { Decreto } 1780 \text { de } 2003 \text { y } \\
734 \text { de } 2004\end{array}$ & $\begin{array}{l}\text { Crea el Premio Colombiano a la Innovación Tecnológica Empresarial para las } \\
\text { MiPyMEs. }\end{array}$ \\
\hline Decreto 4233 de 2004 & $\begin{array}{l}\text { Otorga el Premio Colombiano a la Innovación Tecnológica Empresarial para las } \\
\text { MiPyMEs en el } 2004 \text {. }\end{array}$ \\
\hline Decreto 4484 de 2005 & $\begin{array}{l}\text { Otorga el Premio Colombiano a la Innovación Tecnológica Empresarial para las } \\
\text { MiPyMEs en el } 2005 .\end{array}$ \\
\hline $\begin{array}{l}\text { Resolución } 1021 \text { de } \\
2004\end{array}$ & $\begin{array}{l}\text { Establece el Comité Técnico para el Premio Colombiano a la Innovación } \\
\text { Tecnológica Empresarial para las MiPyMEs }\end{array}$ \\
\hline Decreto 4375 de 2008 & $\begin{array}{l}\text { Otorga el Premio Colombiano a la Innovación Tecnológica Empresarial para } \\
\text { las MiPyMEs en el } 2008 .\end{array}$ \\
\hline
\end{tabular}




\section{La informalidad de las PyMEs: un elemento que afecta el crecimiento y desarrollo económico y social del país}

La insuficiencia de beneficios e incentivos económicos, financieros $y$ tributarios, es una expresión común entre los empresarios; aducen que las cargas tributarias que deben soportar son muy elevadas y que se constituyen en un elemento desfavorable a la subsistencia y crecimiento de sus negocios.

Esta situación trae consigo un hecho preocupante para la sostenibilidad del estado y de las empresas mismas: la informalidad en la que se sumergen éstas y bajo la cual desarrollan su actividad económica, con el pretexto de no percibir el suficiente nivel de ingresos para financiar sus operaciones y para cubrir los tributos; lo que se traduce también en ilegalidad, elemento configurado por los empresarios bajo el argumento de no querer desviar recursos hacia un estado poco benefactor. La informalidad también se refleja en otros aspectos como la no inscripción en el respectivo registro mercantil, carecer de sistemas contables o manejar dobles contabilidades, entre los más principales y notorios, tal como se muestra en el cuadro 3.
Tabla 3

Principales elementos de informalidad en las microempresas colombianas

\begin{tabular}{|l|c|}
\hline Elemento de informalidad & Porcentaje \\
\hline Evasión de impuestos & $53.5 \%$ \\
\hline No llevan registros contables & $42 \%$ \\
\hline No poseen registro mercantil & $45 \%$ \\
\hline
\end{tabular}

Fuente: Documento CONPES 3484 de 2007

Esta situación se ha convertido en una problemática muy generalizada en el país por la cultura propia del mismo y la cual tiene sus repercusiones negativas en su desarrollo económico y social. No puede ocultarse que Colombia se ha caracterizado por la cultura de la evasión (de impuestos, de contribuciones sobre la nómina y de aportes parafiscales, etc.) así como por el individualismo en virtud del cual se pretende conseguir resultados favorables a costa de quien sea o de lo que sea.

Como lo indica Juan Carlos Duran ${ }^{10}$, "la posibilidad de una sanción es vista como un riesgo menor frente al beneficio económico de evadir las obligaciones laborales (prestaciones sociales y contribuciones

10 DURAN, Juan Carlos. La informalidad empresarial. En: http://www.docstoc.com/docs/3253541/La-informalidad-empresarial-ylaboral-en-las-pymes. (Consulta: 18 de agosto de 2009). 
sobre la nómina). Por supuesto, esta ventaja individual para el empresario tiene un enorme costo social que se materializa en la desprotección de sus trabajadores en materia pensional o de salud".

En el mismo sentido, en el país no existe la suficiente conciencia entre los empresarios, de que el pago legal de impuestos contribuye al desarrollo económico de los pueblos porque se traduce en mejores condiciones de vida: infraestructuras (vías, hospitales, planteles educativos...), servicios estatales (educación, salud, recreación, defensa...), servicios públicos, etc. Es más, podría asegurarse que ningún empresario conoce o recuerda el deber constitucional de las personas naturales y jurídicas de contribuir al financiamiento del gasto público del país ${ }^{11}$. Quizá los elevados índices de corrupción que registra el país a nivel de los dirigentes políticos, tampoco favorezca dicha conciencia y dicha situación.

Todo esto hace que el asunto de la informalidad en las empresas se torne complejo y afecte de alguna manera su competitividad, la dinámica y el crecimiento económico del país. El hecho de que los empresarios no se encuentren debidamente organizados origina consecuencias evidentes que se reflejan en situaciones como:

11 Numeral $9^{\circ}$, Artículo 95 de la Constitución Política de Colombia.
$>$ Impedimento en la mejora de sus procesos productivos y por tanto la calidad de los bienes y servicios que ofrecen. Además el ingreso a mercados internacionales es mucho más difícil, por no decir que imposible.

DImposibilidad para acceder a contratos con el estado como cliente principal de las economías de los países, por los requerimientos que este establece $\mathrm{y}$ los cuales no pueden cumplir desde su informalidad.

D Obstaculización para el acceso a recursos del mercado financiero porque no pueden suministrar información sencilla como estados y proyecciones financieras.

> No acceso a algunos beneficios otorgados por el estado en circunstancias especiales en razón a no poder acreditar los documentos y demás requisitos exigidos por este. Un ejemplo, los beneficios por cobertura cambiaria en épocas de constante revaluación del peso.

$>$ Sus procesos de toma de decisiones se tornan complejos por no poseer información para el efecto; y no contar con la compañía y asesoramiento de personal cualificado. Este hecho puede suscitar la toma de decisiones 
desacertadas y el riesgo de decisiones que afecten la sobrevivencia y crecimiento de la empresa; ejemplo: desacertadas decisiones de apalancamiento..

Estos son simples ilustraciones de situaciones que afectan de una u otra manera el desarrollo empresarial, económico y social de un país. La ambivalencia de los empresarios al decidir si operar de manera legal o no, repercute de manera negativa en sus negocios, en la sociedad y en la economía. Luis Fernando Andrade ${ }^{12}$, sintetiza este hecho en que la informalidad "es un obstáculo importante al desarrollo económico del país. Esto ocurre porque el gobierno, al no poder cobrar impuestos a la economía informal, acaba financiando sus actividades con el aumento de impuestos a las empresas formales. Las empresas formales tienen que competir en desigualdad de condiciones con las empresas informales, las cuales gozan de la evasión del impuesto de renta, del IVAy de las contribuciones relacionadas con la nómina de sus empleados. Dependiendo del sector, esto puede representar entre 10 y $30 \%$ del costo de venta de un producto o servicio. La competencia desleal por parte de los informales afecta la capacidad de crecimiento de las empresas formales, que ofrecen empleos de mayor calidad. La productividad del país también se afecta, porque las empresas formales son mucho más productivas que las informales".
Por lo anterior, no pueden terminar en vano las propuestas que se han venido suscitando últimamente y de manera insistente, respecto a trabajar en temas de responsabilidad social empresarial, responsabilidad ambiental de las empresas, ética empresarial, entre otros; como instrumentos que conduzcan a los empresarios a desarrollar sus procesos de manera legal, garantizando su crecimiento y permanencia en el tiempo y en el espacio y contribuyendo de alguna manera a la sostenibilidad, crecimiento y desarrollo de la economía del país, lo que redunda en bienestar social y mejor calidad de vida para todos.

\section{Conclusiones}

C olombia ha experimentado un notable incremento en la creación de nuevas empresas durante los últimos años. Ello, aunque ha permitido dinamizar un poco la economía principalmente a través de la generación de empleo, no ha repercutido de manera significativa en el crecimiento y desarrollo económico y social del país, por cuanto las empresas establecidas y nacientes, no logran fabricar y posicionar productos con agregación de valor, como resultado de procesos de innovación, investigación y desarrollo.

\footnotetext{
${ }^{12}$ ANDRADE, Luis Fernando. El problema de la economía informal. En: http://www.dinero.com/noticias-columnistas/problemaeconomia-informal/31843.aspx. (Consulta: 18 de agosto de 2009).
} 
De otra parte, la estructura empresarial del país está conformada en un porcentaje muy elevado, por microempresas y por PyMEs, hecho que tiene fortalezas, pero también dificultades tales como: limitada capacidad de innovación, limitado acceso a los recursos del mercado financiero, escaso personal cualificado, bajo nivel tecnológico, entre otros. Estas situaciones crean de alguna manera obstáculos para hacer frente a los competidores y por tanto para extenderse y permanecer en el mercado.

Las políticas y estímulos para fomentar la actividad emprendedora en el país, no han logrado dar los resultados esperados; por lo tanto deben ser fortalecidas y prolongadas en eltiempo; sólo asíse lograrán consolidar procesos empresariales adecuados a las necesidades del mercado nacional e internacional con innovación tecnológica.

La informalidad de los negocios, reflejada en evasión y otras prácticas ilegales, además de afectar su propio desarrollo empresarial, conlleva a resultados funestos para el estado quien ve reducidas sus bases impositivas y por tanto sus recaudos, con lo que limita su capacidad de inversión y compensación hacia la sociedad, y con ello la continuidad de un circulo vicioso en el que nunca se logran evidenciar índices adecuados de crecimiento y desarrollo económico, mejores condiciones de vida de la población, etc.

\section{Referencias bibliográficas}

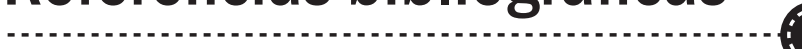

Andrade, L. (2009). El problema de la economía informal. En: http://www.dinero.com/ noticias-columnistas/problema-economia-informal/31843.aspx.

Calle, A. et.al (2009). Estrategia, competitividad y cambio organizacional en el proceso de internacionalización de las PyMEs. En: www.eafit.edu.co/.../ CompetitividadInternacionalizacionCambio.doc. CONGRESO DE LAREPUBLICA. Ley 1014 de enero 26 de 2006. "De fomento a la cultura del emprendimiento".

Congreso de la República. Ley 1111 de 2006 de diciembre 27 de 2006. "Por la cual se modifica el estatuto tributario de los impuestos administrados por la dirección de impuestos y adunas nacionales". 
Congreso de La República. Ley 590 de 2000. "Por la cual se dictan disposiciones para promover el desarrollo de las micro, pequeñas y medianas empresa”. Artículo 43.

Congreso de La República. Ley 905 de 2004 (Diario oficial No 45.628 del 02 de agosto de 2004). "Por medio de la cual se modifica la Ley 590 de 2000 sobre promoción del desarrollo de la micro, pequeña y mediana empresa colombiana y se dictan otras disposiciones".

Consejo Nacional de Política Económica y Social. Documento CONPES 3484 de 2007 (Política nacional para la transformación productiva y la promoción de las micro, pequeñas y medianas empresas. Un esfuerzo público privado).

Constitución Política de Colombia

Duran, J. (2009). La informalidad empresarial. En: http://www.docstoc.com/docs/ 3253541/La-informalidad-empresarial-y-laboral-en-las-pymes.

Granados H. (2009). El papel de las micro y pequeñas empresas en el desarrollo territorial: Una lectura desde los programas de fortalecimiento empresarial en la ciudad de Medellín. En: Mercatec, revista de la Institución Universitaria Esumer. No 45,. Pág. 20 a 31.

Informe GEM Colombia 2007. Reporte de Resultados.

Informe GEM Chile 2008

Ministerio de Comercio, Industria y Turismo. (2009). Importancia y dificultades de las MIPYMES. En: http://www.mincomercio.gov.co/eContent/NewsDetail.asp?Source=Ex piredNews\&ID=1294\&IDCompany=1.

Varela R. (2008) Innovación Empresarial, Arte y Ciencia en la Creación de Empresas. Bogotá: Pearson Prentice Hall. 646 p. 